\title{
Online Optical Tomography System Application of Charge-Coupled Device (CCD) for Object Detection in Crystal Clear Water
}

\author{
Juliza Jamaludin ${ }^{1,2}$, Ruzairi Abdul Rahim ${ }^{1}$, Herlina Binti Abdul Rahim ${ }^{1}$, \\ Mohd Hafiz Fazalul Rahiman ${ }^{3}$, Siti Zarina Bte. Mohd Muji ${ }^{4}$, Jemmy Mohd Rohani ${ }^{5}$ \\ ${ }^{1}$ Process Tomography and Instrumentation Engineering Research Group (PROTOM-i), \\ Infocomm Research Alliance, Faculty of Electrical Engineering, \\ Universiti Teknologi Malaysia, 81310 UTM Johor Bahru, Johor, Malaysia. \\ ${ }^{2}$ Faculty of Engineering and Built Environment, Universiti Sains Islam Malaysia, \\ 71800 Bandar Baru Nilai, Negeri Sembilan, Malaysia. \\ ${ }^{3}$ Tomography Imaging Research Group, School of Mechatronic Engineering, \\ Universiti Malaysia Perlis, 02600Arau, Perlis, Malaysia. \\ ${ }^{4}$ Faculty of Electrical and Electronic Engineering, Universiti Tun Hussein Onn Malaysia, \\ 86400Parit Raja, Batu Pahat, Johor Malaysia. \\ ${ }^{5}$ Sondotech Sdn. Bhd, 31, Jalan Mutiara Emas 5/1, Taman Mount Austin, 81100, Johor, Malaysia. \\ 'juliza.hj.jamaludinegmail.com \\ ${ }^{2}$ ruzairi@fke.utm.my \\ ${ }^{3}$ herlinalfke.utm.my \\ ${ }^{4}$ hafizeunimap.edu.my \\ ${ }^{6}$ jemmymohdrohani@gmail.com
}

\begin{abstract}
This research presents an application of Charge-Coupled Device (CCD) linear sensor and laser diode in an optical tomography system. These optoelectronic sensors are believed to detect solid objects rather than transparent objects. Based on the research results, the development of optical tomography system using CCD and laser diode has helped to enhance the potential of these sensors in detecting low opacity objects. Experiments for detecting a solid rod, transparent rod and air bubbles in non-flowing crystal clear water were conducted in this study. From the image reconstruction results, it clearly shows a cross-sectional image of a pipeline system with the existence and the exact location of opaque and transparent objects in multiphase flow detected by the orthogonal projection of CCD sensors and lasers. The information details of real-time multiphase flow that observed by this system can be transmit via wireless or wired data communication system.
\end{abstract}

Keywords -Charge-Coupled Device (CCD), laser diode, optical tomography system, image reconstruction, multiphase flow

\section{INTRODUCTION}

Underwater data communication is very important nowadays to retrieve the information of foreign object that exists in liquid medium. The applications of underwater data communication system are high in demand for oil industries to marine life [1]. Traditional technique known as acoustic sensor array has a limitation when dealing with real-time operation because single sampling rate and synchronization errors can occur by the distribution of different hydrophones [2]. Meanwhile, unmanned autonomous vehicles (AUVs) and autonomous robots are used for underwater data communication [3] based on video cameras high in cost and suitable for vast monitoring processes such as marine environment.

Industries that deal with multiphase flow also require an underwater monitoring system that can detect solid or transparent objects in liquid medium but at a small scale. CCD sensor has high sensitivity and accuracy, high frame rates, wide dynamic range and low power consumption $[4,5]$. The basic principle of CCD is to measure the level of light intensities received by its small sensors, which are made from 
semi-conductor components [6]. As a result, CCD is believed to be able to detect opaque objects only. This is because opaque objects will reflect or absorb the light that hits their surface [7]. The final amount of light intensities received by CCD will determine the existence and position of the object. This phenomena show that CCD has high sensitivity to detect dark spots only $[8,9]$.

CCD is mostly applied as an optical displacement sensor $[10,11]$, surface detection sensor [12], thickness detection sensor [13] and object detection system using a CCD camera [14]. Z. Fei et al. [14] concluded that laser and CCD cameras can be the best combination because CCD cameras have high precision for 2D image measurement while laser provides high precision in the axial direction. The use of laser gives high light illumination, wide range of measurement and superior resolution of image. For research done by Yang Ni et al. [13], the combination of CCD and laser have helped to construct thickness detection tools that lead to high sensitivity, precise accuracy and high stability in reading distance. These circumstances proved that CCD and laser diode are the best transceivers for opaque object measurement technologies. For transparent objects, the light source can penetrate more compare to solid objects. This may make it difficult for CCD to detect transparent objects because light intensities received by CCD may not differ significantly.

In this context, a research has been conducted to solve the mentioned problem by applying the optical tomography approach [15]. Firstly, this project has optimized the use of the above sensors in capturing and measuring a cross-sectional image of a non-invasive pipeline system by expanding the laser diode light source, with the assumption that the light expansion method will help these transceivers widen the coverage area.

Secondly, CCD linear sensors and laser diodes have been oriented in an orthogonal projection to enhance the image reconstruction of low opacity objects. The overlapping of the expansion light beam received by CCD sensors will help the detector determine the exact location and appearance of low opacity objects. Details on hardware construction are explained in the methodology section.

For image reconstruction, Linear Back Projection (LBP) algorithms have been used. This LBP image has been enhanced where the LBP object detection image result has been filtered by the LBP initial image result. The LabVIEW software has been used for image reconstruction and analysis. Further, analysis in comparing the images of these three different objects is presented in this paper.

\section{METHODOLOGY}

There are two types of CCD architecture: area array $\mathrm{CCD}$ and linear $\mathrm{CCD}$. This project focuses on the line scan camera or linear CCD because it is small in size and consists of a single line photosensitive element [16]. As a result, linear CCD provides less scan time per frame compared to array CCD [16]. This research uses CCD linear sensor Sony ILX551A [17]. Grey CCD (Sony ILX551A) has been chosen because its time scan rate ratio is 1:3 times lower than color CCD [18]. Color CCD consists of red, green and blue photosensitive elements and needs a large memory for saving output signals [19]. This type of CCD sensor can detect photon spectral range of 400 to $1100 \mathrm{~nm}[20,21]$. For this flow measurement instrument, we chose low cost laser diode with a mixture of Helium and Neon gases in the ratio of 10:1. It is an atomic laser with low power device [22]. This type of laser diode emits photons with spectral range of $633 \mathrm{~nm}$ to $650 \mathrm{~nm}$ and is suitable for the Sony ILX551A CCD linear sensor. The sensitivity peaks that are detected by CCD sensors are normally in the range of 550 to $800 \mathrm{~nm}$ [20].

As mentioned before, the main purpose of this hardware development is to inspect the existence of different levels of opacities of objects in crystal clear water. Crystal clear water helps in absorbing heat that is produced by laser diode, thereby preventing overheating and causing damage to the CCD sensors. Besides that, high thickness of water level (refer to diameter of the pipeline system) can provide a long pathway that the laser has to undergo, causing the heat affected zone (HAZ) of the laser diode to become less significant [23]. Four pairs of CCD sensors and laser diodes expansion system were arranged in orthogonal projection, as shown in Figure 1. This orthogonal projection helps the sensors to uniformly view the cross-sectional image of the flow system. The diagram of the laser diode expansion system box is shown in Figure 2.

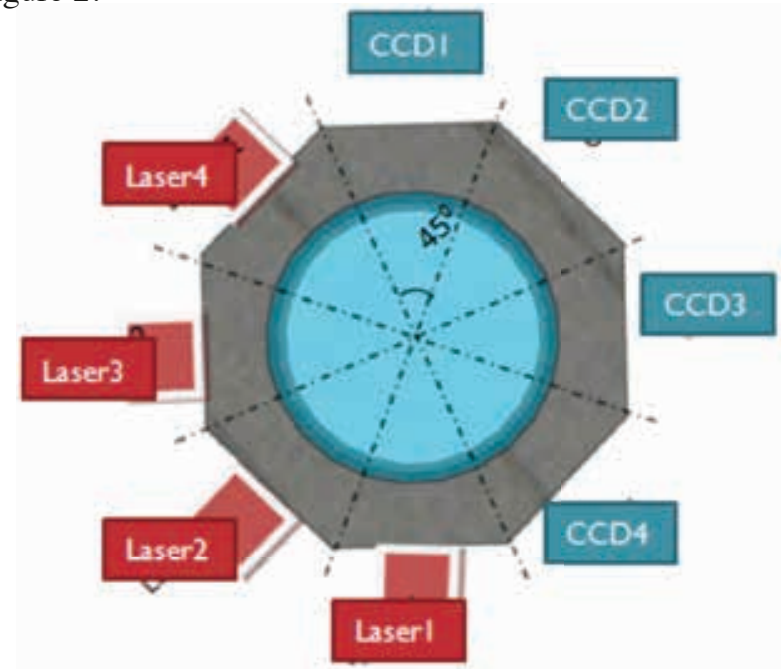

Fig.(1). Sensor orientation 


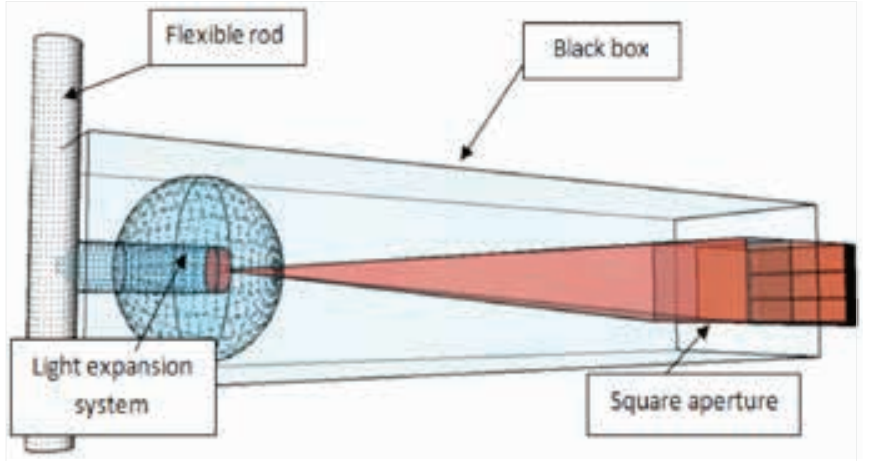

Fig.(2). Laser diode expansion system box

The square aperture dimension of $40 \mathrm{~mm} \times 10 \mathrm{~mm}$ were aligned parallel with its CCD sensor. A lens with a focal length of 0.5 was attached at the front of the laser diode source. The laser diode light source expanded into a fan beam projection form. The expansion light source is then passed through the square aperture. This square aperture was used to limit the area of the laser diode beam that can reach the CCD surface sensor. The final light source received by the CCD sensor is known as the square beam projection. In Figure 3, the illustration explains the light transmitting process from the laser diode source to the CCD sensor.

To obtain an accurate light source expansion, a laser diode was fixed to an adjustable rod so that the distance between the laser and CCD can be controlled manually. The CCD frame was used to fix the CCD sensors at a desirable level of the pipeline system. This frame provided six sockets for the CCD sensor to be attached at each column. However, this experiment used only one CCD socket. The pipeline and sensor system were built in a closed black box to avoid the interruption of an external and visible light source. The hardware construction diagram can be seen in Figure 4. The lux received by each CCD sensor was measured as 0.3 lux value.

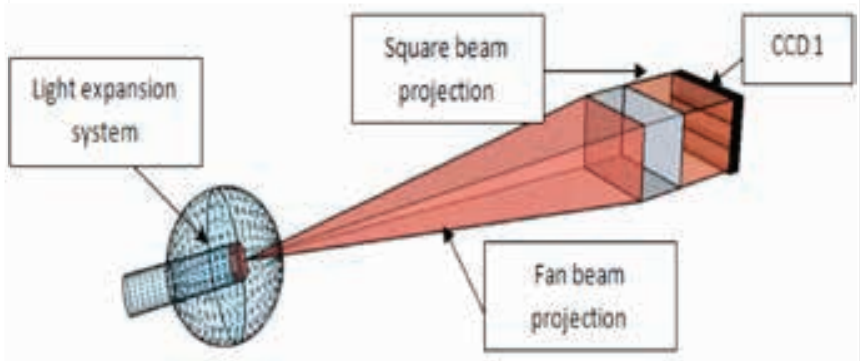

Fig.(3). Light transmitting process

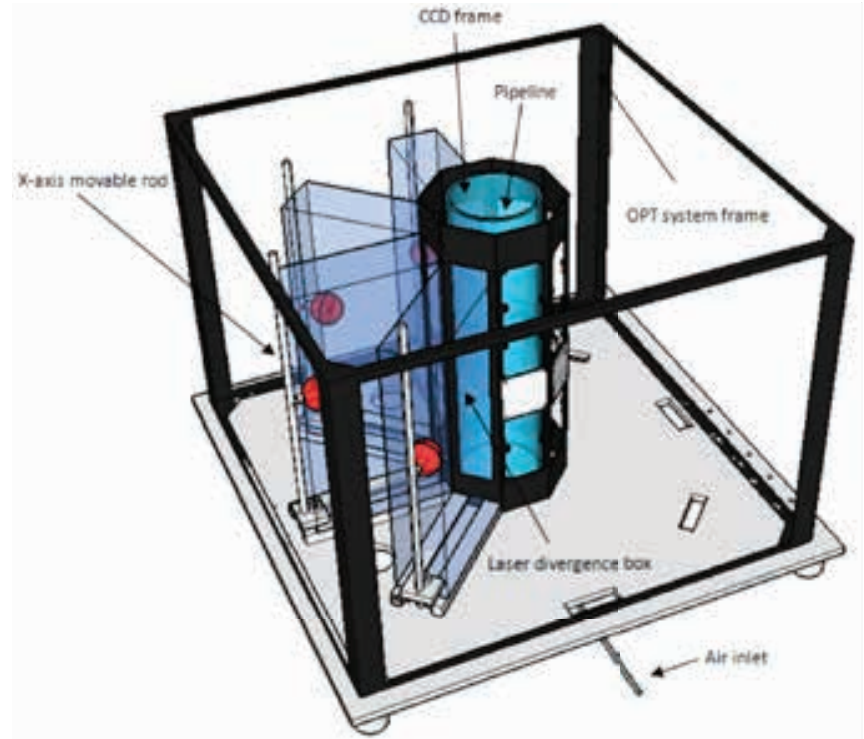

Fig.(4). Hardware construction schematic diagram

Meanwhile, Figure 5 shows the final results of the optical tomography system using CCD linear sensor as a detector with the observation pipeline system which were built together in a black box. Data acquisition NI USB-6210 was used for the interfacing process between the hardware and software construction.

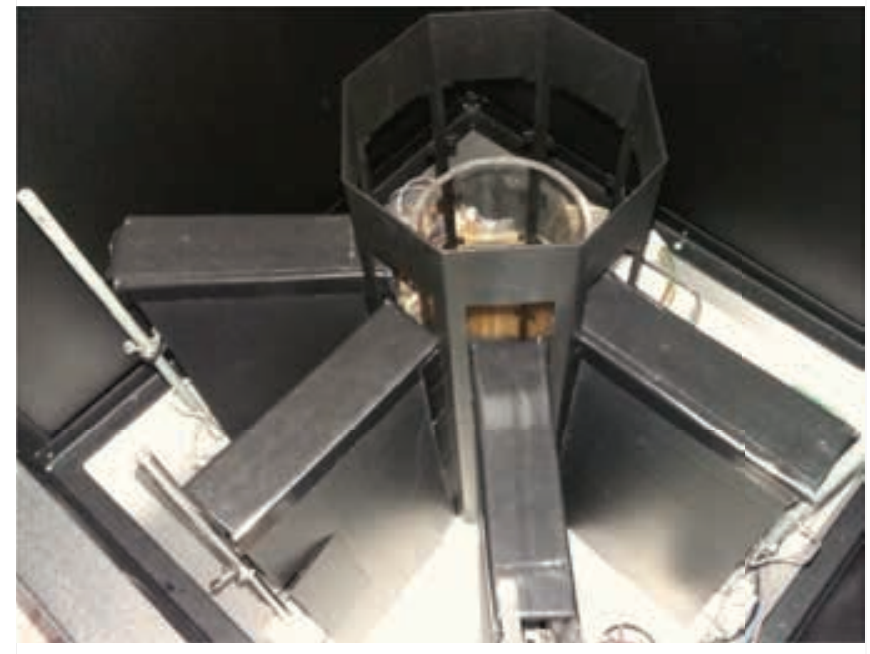

Fig.(5). Optical tomography system using CCD sensor

The CCD linear sensor Sony ILX551A requires two signals: Read Out Gate (ROG) and a clock pulse generator to function, with both signals programmed using $\mathrm{C}$ language in PIC16F877A. For the clock pulse, the time per cycle was 8.80 $\mu \mathrm{s}$ and total time scan for this optical tomography system was $16 \mathrm{~ms}$ per frame $[24,25]$.

Development of the Labview software for CCD image reconstruction involved in this study. The Labview front 
panel for software program of this optical tomography system is shown in Figure 6.

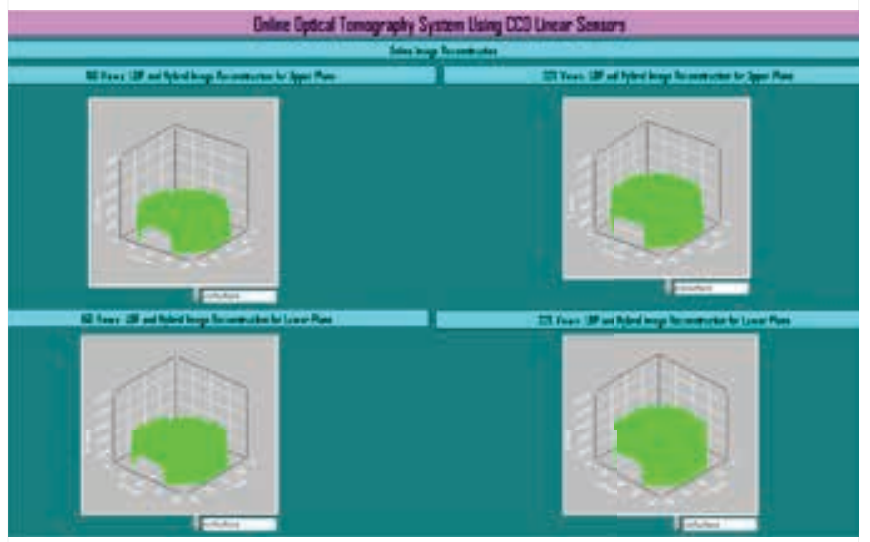

Fig.(6). Labview front panel for optical tomography system using CCD sensor

Sensitivity maps for a $100 \mathrm{~mm}$ diameter acrylic pipeline with 160 and 320-views in $40 \mathrm{~mm}$ length of the sensor were developed using available Visual Basic software. Each sensitivity map was multiplied by the CCD voltage output for image reconstruction. Resulting images of the solid rod, transparent rod, and air bubbles in static clear water were analysed to prove that CCD sensors are able to detect different opacities of objects using the optical tomography method. The results and discussion section will explain in detail about the performance and achivement of this optical tomography system hardware development.

\section{RESULTS AND DISCUSSION}

Several experiments were carried out in detecting a solid rod, transparent rod and air bubble in non-flowing crystal clear water. All the calculations were obtained using the matrix form. The LabVIEW software transformed the matrix results into a color map, also known as an image reconstruction for the optical tomography system. The color map values in fact were a result of changes in light intensities received by the CCD sensors. These light intensities are considered as analogue signals which are converted into voltage values or digital signals using the NI USB-6210 data acquisition system. The data generated are then used in the LabVIEW programming for image reconstruction.

Figure 7 and Figure 8 show the orthogonal image reconstruction results of (a) solid rod, (b) transparent rod and (c) air bubble in static clear water for 160 and 320-views. The image shown in Figure 7 and Figure 8 respectively was obtained using the LBP algorithm. (a).

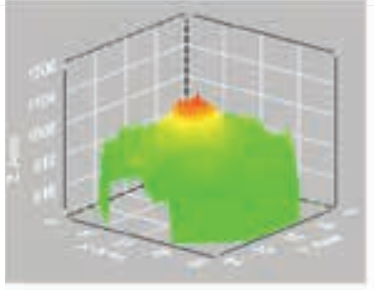

(b).

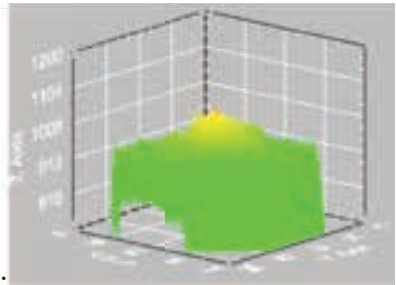

(c).

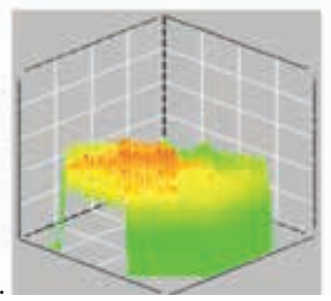

Fig.(7). (a) Solid rod, (b) Transparent rod and (c) air bubbles in 160-views

(a).

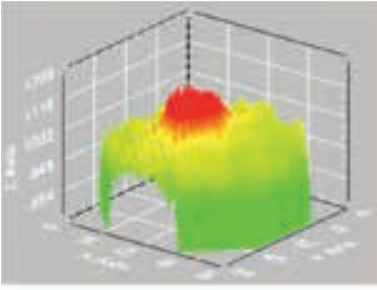

(b).

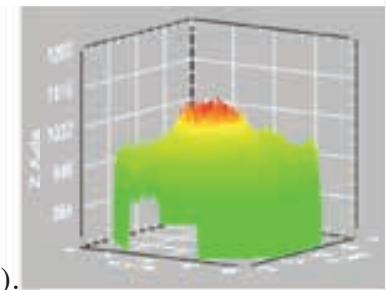

(c).

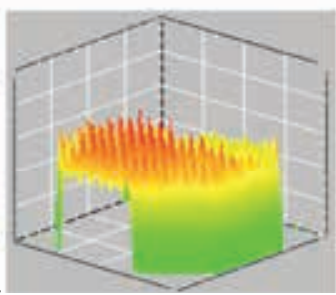

Fig.(8). (a) Solid rod, (b) Transparent rod and (c) air bubbles in 320-views

Equation (1) was applied to get the above LBP image reconstruction results.

$[$ Final Image $(\mathrm{LBP})]=[\text { Normalized Sensitivity map }]^{*} \mathrm{CCD}$ Voltage Output....

Then, equation (2) was applied to enhance the LBP image reconstruction results.

$[$ Filtered image $]=[$ Final image $(\mathrm{LBP})]-[$ Initial image (LBP)]

The initial image in (2) refers to the initial state of the pipeline system without any object inside it. Figure 9 and Figure 10 show the results of three different opacities objects using equation (2). 
The LBP image results shown in Figure 7 and Figure 8 prove that CCD sensors can detect the existence of solid and transparent objects. It should be noted that images in Figure 9 and Figure 10, show the appearance and location of the static solid rod to be clearer than the static transparent rod because more light can penetrate through transparent objects compared to solid objects.

(a).
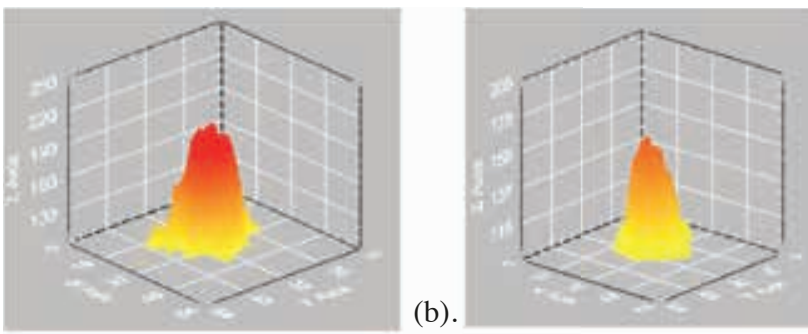

(c).

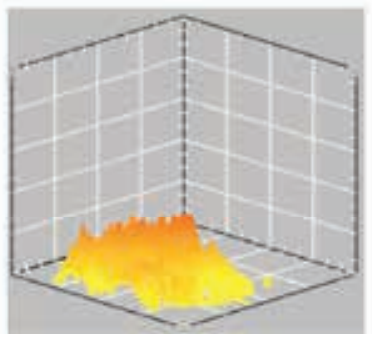

Fig.(9). (a) Solid rod, (b) Transparent rod and (c) air bubbles in 160 -views for filtered images.

(a).
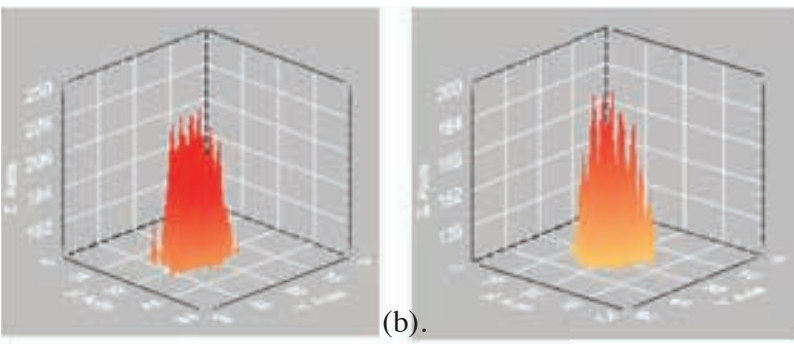

(c)

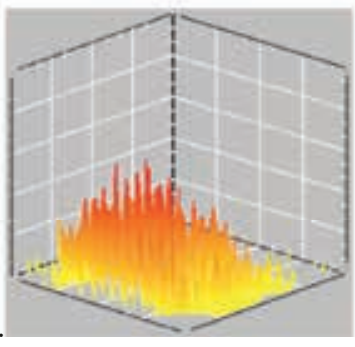

Fig.(10). (a) Solid rod, (b) Transparent rod and (c) air bubbles in 320-views for filtered images

Theoretically, the reconstructed images of the air bubbles should be less clear, compared to static solid and transparent objects due to the low opacity of the air bubbles.
Air bubbles with a volume of $20 \mathrm{ml}$ were produced using a syringe. Thus, the shape and distribution of the bubbles produced were not controllable. Most of the produced bubbles have a larger diameter or are scattered in a large area.

Besides that, image reconstruction for 320-views gives a higher resolution of object distribution compared to 160 -views. This non-invasive, non-intrusive and radiation free hardware has a bright future in the monitoring system of the multiphase flow industry.

\section{CONCLUSION}

The application of CCD sensors and laser diode in an optical tomography may enhance the potential of these sensors in capturing a cross-sectional image from solid to transparent objects. This study also proves the ability of CCD sensors to measure the cross-sectional position of objects, in a multiphase pipeline system.

\section{ACKNOWLEDGMENT}

The authors would like to thank to Universiti Teknologi Malaysia and Universiti Sains Islam Malaysia for supporting the research study and PROTOM research group for their cooperation in this research paper.

\section{REFERENCES}

[1] J. Heidemann, M. Stojanovic and M. Zorzi, "Underwater sensor networks: applications, advances and challenges," Philosophical Transactions of the Royal Society A: Mathematical, Physical and Engineering Science, vol. 370, pp: 158-175, 2012.

[2] Y. Li and F. A. J. J. Duan, "Underwater acoustic sensor array based on variable sampling rates and high precision synchronization," Sensors and Actuators A: Physical, vol. 242, pp: 99-105, 2016.

[3] Akyildiz and P. A. L. S. Wang, "SoftWater: Software-defined networking for next-generation underwater communication systems," Ad Hoc Networks., vol. 46, pp: 1-11, 2016.

[4] G. He, X. Wang, D. Li and A. J. Hu, "A High Speed Image Sensing Technique with Adjustable Frame Rate Based on an Ordinary CCD," Optik, vol. 119, pp: 548-552, 2008.

[5] L. Gang, S. H. Bin, L. Li, Z. Chu, M. S. Juan and W. Y. Bo, "Laser Induced Damages to Charge Coupled Device Detector Using a High Repetation Rate and High Peak Power Laser," Optics and Lasers Technology, vol. 47, pp: 221-227, 2012.

[6] J. Fraser, D. H. Alexander, C. R. M.Finnila and S. C. $\mathrm{Su}$, "An Extrinsic Silicon Charge Coupled Device for Detecting Infrared Radiation," In Proceedings of Electron Devices Meeting (IEDM), 1974. 
[7] K. Gangulay, "Wave Particle Duality of Light," in Optical and Optoelectronic Instrumentation, Calcutta University, Alpha Science International Limited, 2010, pp: 1-16.

[8] S. Z. M. Muji, C. L. Goh, N. Ayob, R. A. Rahman, M. H. F. Rahiman, H. A. Rahim, M. J. Pusppanathan and N. S. M. Fadzil, "Optical Tomography Hardware Development for Solid Gas Measurement Using Mixed Projection," Flow Measurement and Instrumentation, vol. 33, pp: 110-121, 2013.

[9] Buil, "The Electronics of a CCD Camera," in Charged Coupled Device : Astronomy, Virginia, Willmann Bell Inc, 1991, pp: 56-57.

[10] Q-P. Li, F. Ding and P. Fang, "Flash CCD Laser Displacement Sensor," Electronics Letter, vol. 42, no. 16, pp: 910-912, 2006.

[11] Z. Hong, W. Xuan and W. Rui, "High Speed on Line Measurement of Digital Wire Outer Diameter with Laser and CCD Technology," In Proceedings of the $7^{\text {th }}$ International Conference on Properties and Applications of Dielectrics Materials, Nagoya, Japan, 2003.

[12] Zhou, J. Guo and W. Shao, "Automated Detection of Surface Defects on Sphere Parts Using Laser and CCD Measurement," In Proceedings of $37^{\text {th }}$ Annual Conference on IEEE Industrial Electronics Society (IECON), 2011.

[13] Y. Ni, W. Y. Tian, L. J. Tao and L. H. Huan, "Research on Thickness Measurement of Transparent Object Based on CCD," In Proceedings of International Conference on Measuring Technology and Mechatronics Automation, 2009.

[14] Z. Fei, J. Guo, J. Wang and G. Luo, "The Application of Laser and CCD Compound Measuring Method on 3D Object Detection," In Proceeding of the International Conference on Mechatronic and Automation, Xian, China, 2010.

[15] P. Grangeat, Digital Signal and Image Processing Series: Tomography, Great Britain: John Wiley \& Sons, 2009.

[16] J. Lenart, "Optical Vibration Analysis with Linear CCD Sensor and Reconfigurable Hardware," Procedia Engineering, vol. 96, pp: 268-272, 2014.
[17] "Data Sheet for ILX 551A", Sony, Japan.

[18] S. Kawamato, Y. Watanabe, Y. Otsuka and T. Narabu, "A CCD Color Linear Image Sensor Employing New Transfer Method," Consumer Electronics, IEEE Transactions, vol. 37, no. 3, pp: 481-486, 1991.

[19] T. G. Etoh, D. Poggemann, G. Kreider, H. Mutoh, A. J. Theuwissen, A. Ruckelshausen and Y. Kondo, "An Image Sensor which Captures 100 Consecutive Frames at 1000000 Frames/s," IEEE Transaction on Electron Devices, vol. 50, no. 1, pp: 144-151, 2003.

[20] K. R. Spring, T. J. Fellers and M. W. Davidson. (2013). "Nikon: The Source for Microscopy Education," [Online]. Available: https://www.microscopyu.com/articles/digitalimaging/ccdintro.html. [Accessed $29 \quad 5$ 2015].

[21] J. Madigan. (2011). "NASA," National Aeronautic and Space Administration, [Online].Available:http://science-edu.larc.nasa.gov/

EDDOCS/Wavelengths_for_Colors.html. [Accessed 31 5 2015].

[22] K. Gangulay, "Laser," in Optical and Optoelectronic Instrumentation, Asansol, India, Alpha Science International LTD, 2010, pp: 7.1-7.32.

[23] H. Shen, M. Ran, J. Hu and A. Z. Yao, "An Experimental Investigation of Underwater Pulsed Laser Forming," Optics and Lasers in Engineering, vol. 62, pp. 1-8, 2014.

[24] J. Jamaludin and R. A. Rahim, "Online Optical Tomography System for Detecting and Measuring the Diameters of Solid and Transparent Objects," IEEE Sensor Journal, vol. 16, no. 16, p. 6175-6183, 2016.

[25] J. Jamaludin, R. A. Rahim, H. A. Rahim, M. H. F. Rahiman and S. Z. M. Muji and J. M. Rohani. "Charge Coupled device based on optical tomography system in detecting air bubbles in crystal clear water," Flow Measurement and Instrumentation, vol. 50, 2016.

(C) Author(s) 2016. CC Attribution 4.0 License. (http://creativecommons.org/licenses/by-nc/4.0/)

This article is licensed under the terms of the Creative Commons Attribution Non-Commercial License which permits unrestricted, non-commercial use, distribution and reproduction in any medium, provided the work is properly cited. 